\title{
Numerical Simulations of Natural and Actuated Flow over a 3D, Low-Aspect-Ratio Airfoil
}

\author{
Guillaume A. Brès*, \\ Exa Corporation, Brisbane, CA 94005 \\ David R. Williams ${ }^{\dagger}$ \\ Illinois Institute of Technology, Chicago, IL 60616 \\ and \\ Tim Colonius ${ }^{\ddagger}$ \\ California Institute of Technology, Pasadena, CA 91125
}

\begin{abstract}
Numerical simulations of the unsteady flow over a low-aspect-ratio, low Reynolds number semi-circular planform wing are performed using Lattice Boltzmann method. The simulations exactly match the flow conditions and the detailed geometry from previous wind-tunnel experiments, including the flow actuators installed internally along the leading edge of the wing. To reproduce the pulsed-blowing actuation used in the experiment, a single pulsed square wave forcing is imposed in the simulations as a mass flow boundary condition in the actuators. Three angles of attack, with the active flow control both on and off, are investigated. For both mean and unsteady lift and drag, the numerical simulations show good agreement with the experiments. In particular, the transient increase in lift after the forcing is turned off is well captured in the simulations. Both PIV measurements and transient numerical results indicate that this behavior is associated with the advection of large vortical structures generated by the flow actuation at the leading edge.
\end{abstract}

\section{Nomenclature}

$\begin{array}{ll}c & \text { Chord } \\ D & \text { Drag } \\ L & \text { Lift } \\ R e & \text { Chord Reynolds number } \\ S & \text { Planform area } \\ t & \text { Time } \\ U_{0} & \text { Freestream velocity } \\ u, v, w & \text { Cartesian velocity components }\end{array}$

$\begin{array}{ll}x, y, z & \text { Streamwise, spanwise and normal directions } \\ \Delta L & \text { Transient change in lift } \\ \nu & \text { Kinematic viscosity } \\ \rho & \text { Density } \\ \alpha & \text { Angle of attack } \\ \omega & \text { Vorticity vector }\end{array}$

\section{Introduction}

In the past decade, there has been significant interest in a new class of smaller unmanned aerial vehicle (UAV), called micro-air vehicles (MAV), for military and law-enforcement purposes. These MAVs are typically one or two orders of magnitude smaller and lighter than traditional UAV, and designed for short autonomous flights, to perform close-range reconnaissance and data-gathering missions. ${ }^{1}$ Despite the sustained research effort, the design and development of efficient MAV remain challenging. The combination

*Principal Aeroacoustics Engineer; Senior Member AIAA

$\dagger$ Professor, Dept. of Mechanical Materials and Aerospace Engineering; Associate Fellow AIAA

${ }^{\ddagger}$ Professor, Dept. of Mechanical Engineering; Associate Fellow AIAA 
of low-aspect-ratio wings (typically $A R<2$ ) operating at relatively low Reynolds numbers (in the range of approximately $710^{4}$ to $210^{5}$ ) is a major challenge, in particular for numerical studies. As a results, most of the computational work on MAV has focused on simpler two- and three-dimensional planform geometries operating at lower Reynolds numbers, ${ }^{2,3}$ with emphasis on flow control. ${ }^{4}$ While these studies have significantly improved the understanding of the fundamental flow physics, a need for simulations of the fully detailed MAV in real flight conditions still exists.

This paper presents a computational study aimed at simulating the unsteady flow over a semi-circular planform wing including the effect of leading edge blowing. The model is based on the experimental results by Williams et al.,$^{5-8}$ within the scale of a full-size MAV. The experiment allows for active flow control through the use of sixteen micro-valves for pulsed-blowing actuation, along the leading edge of the wing. Both natural (i.e., actuation off) and actuated flows are considered in this computational study. A brief overview of the experimental setup is presented in section II.

Numerical simulations of the same configuration are performed using an unsteady fluid flow solver based on the Lattice Boltzmann method (LBM). The Lattice Boltzmann method is a well-known computational fluid dynamics (CFD) method that models flow in a way that is consistent with formal solutions of the transient, viscous and compressible Navier-Stokes equations. ${ }^{9-11}$ The Lattice Boltzmann scheme together with the large eddy turbulence model was shown to accurately capture the aerodynamics of high Reynolds number flows, ${ }^{12,13}$ as well as turbulent wall pressure fluctuations due to separated and reattached flows. ${ }^{14,15}$ As a result, LBM is now being widely used as an investigative and prediction tool for simplified geometries, ${ }^{13,16,17}$ as well as practical engineering applications in the automobile ${ }^{18,19}$ and aerospace ${ }^{20-22}$ industries. Details on the LBM scheme and numerical setup are presented in section III.

The LBM simulations exactly match the flow conditions and the detailed geometry (including the actuators) in the experiment. Different angles of attack, with the active flow control both on and off, are investigated. The results for the natural and actuated flows are discussed in section IV.

\section{Experimental configuration}

The test model used in this study is a low-aspect-ratio, low Reynolds number semicircle airfoil mounted on a vertical sting in a wind tunnel. ${ }^{5,6}$ Schematics of the wing and the Andrew Fejer Unsteady Flow Wind Tunnel are shown in figure 1.

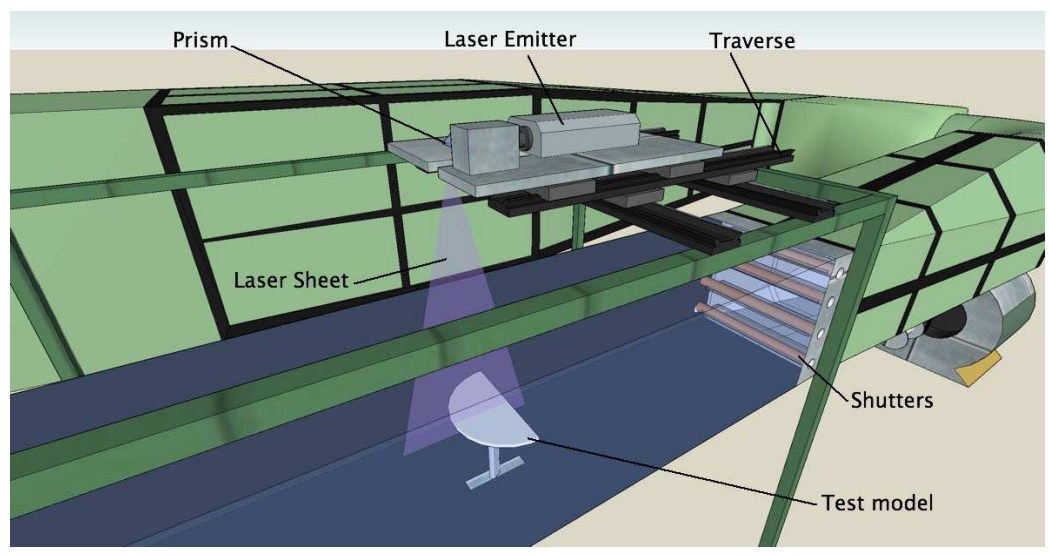

Figure 1. Schematic of the Andrew Fejer Unsteady Flow Wind Tunnel. Flow is from left to right. The test model is the semi-circular planform wing in center of the test section.

The airfoil centerline chord (and radius) is $c=203 \mathrm{~mm}$, such that the span is $b=2 c$, the thickness is $h=0.069 c$, and the aspect ratio is $A R=b^{2} / S=2.54$. The freestream velocity is $U_{0}=5 \mathrm{~m} / \mathrm{s}$, resulting in a chord Reynolds number of $R e=U_{0} c / \nu=68000$. Free stream speed modulation at frequencies of up to $3 \mathrm{~Hz}$ is enabled by a computer controlled shutter at the downstream end of the test section. The model is mounted on a pitch-plunge mechanism, actuated by servotubes. The test section dimensions are $0.6 \mathrm{~m}$ by $0.6 \mathrm{~m}$ by $3.1 \mathrm{~m}$. The models height, pitch angle and pitch rate are regulated, using sting actuation and feedback control. This configuration enables to simulate complex flight maneuvers in captive trajectories. 
The means for active flow control are provided by sixteen micro-valves for pulsed-blowing actuation, installed internally along the leading edge of the wing, as shown in figure 2. All actuators are driven in phase. Real time, on-board sensing is provided by two surface pressure taps at $x / c=0.4$ and $x / c=0.72$. The transient response of the leading edge vortex and the tip vortex system is documented by recording the two surface pressure signals, and the lift, drag and pitching moment using an ATI force 6-component balance system.

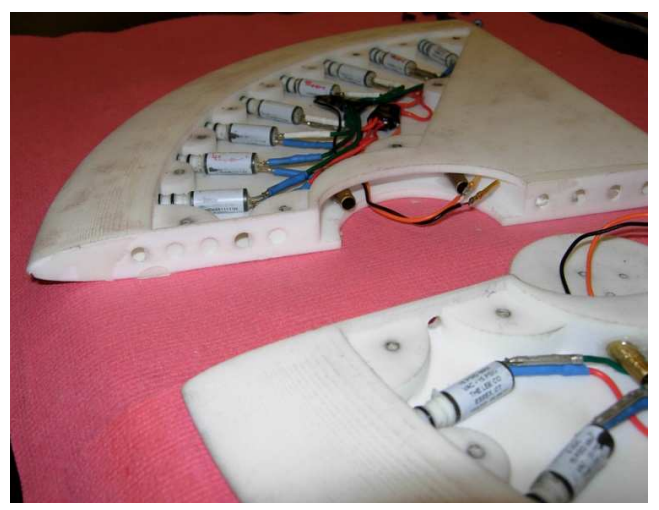

Figure 2. Experimental setup of the sixteen micro-valves for pulsed-blowing actuation

Previous experimental studies ${ }^{4,7}$ have demonstrated the beneficial effect of continuous actuation on the steady lift. While the wing stalls at $\alpha \approx 14^{\circ}$, both with and without actuation, much higher lift values are obtained with the active flow control, for post-stall angles up to $\alpha \approx 20^{\circ}$. The smoke visualization photograph in figure $3(b)$ shows the reattached flow pattern that produces a high lift with the open-loop continuous forcing, compared to the baseline flow without actuation in figure $3(a)$. Here, the actuators were running continuously at $29 \mathrm{~Hz}$.

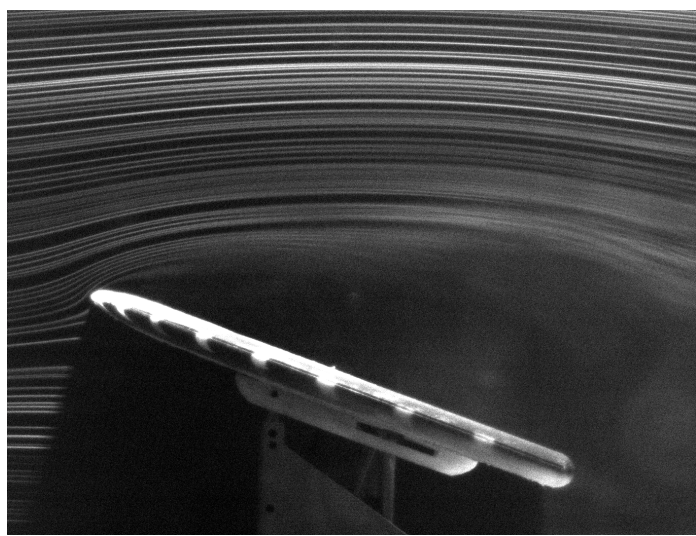

(a)



(b)

Figure 3. Smoke wire visualization of flow over the mid-span of the wing; $(a)$ no forcing; (b) continuous pulsed-jet actuation at $29 \mathrm{~Hz}, 5 \mathrm{psig}$

Similarly, the lift increment due to a single pulsed square wave actuation was studied in recent experimental work. ${ }^{8}$ As a first step and to limit the computational cost, the present numerical study focuses on such short actuator pulse, rather than continuous forcing. In the experiments, the micro-valves are open for $0.017 \mathrm{~s}$, with a constant total mass flow rate of $1.8810^{-3} \mathrm{~kg} / \mathrm{s}$ (summed for all sixteen actuators). The lift and drag forces acting on the semi-circular wing are recorded, with and without leading edge actuation, for angle of attack $\alpha=0^{\circ}$ to $30^{\circ}$. The total duration of the measurements is approximately $1 \mathrm{~s}$. Two-dimensional PIV measurements of the flow field in the mid-span plane are also performed, for comparison with the simulation results. 


\section{Numerical Simulations}

\section{A. Lattice Boltzmann methods}

The CFD code PowerFLOW 4.2 based on the Lattice Boltzmann method is used in this study. Unlike traditional methods solving the discretized Navier-Stokes equations on a computation grid, the LBM approach focuses on the mesoscopic scales via the discrete Boltzmann equation. That is, instead of studying the global behavior of a fluid, LBM tracks the statistical displacement of groups of particles and deducts the macroscopic scale behavior by evolving the particle distribution on a lattice.

The method is briefly reviewed here. The starting point is the distribution function $F_{i}$ expressing the probability of presence of particles at position $\mathbf{x}$ and discrete velocity $\xi_{i}$ at time $t$. The Lattice Boltzmann equation is typically written in the following form: ${ }^{23}$

$$
F_{i}\left(\mathbf{x}+\xi_{i} \Delta t, t+\Delta t\right)-F_{i}(\mathbf{x}, t)=\frac{\Delta t}{\tau}\left[F_{i}^{e q}(\mathbf{x}, t)-F_{i}(\mathbf{x}, t)\right] .
$$

The left-hand side of equation (1) corresponds to the particle advection, while the right-hand side is the collision term, representing relaxation (i.e., interaction of the particles). The well-known BGK approximation $^{24}$ is used to compute the local equilibrium distribution function $F_{i}^{e q}(\mathbf{x}, t)$. The definition of the relaxation time $\tau$ is related to the kinematic viscosity and temperature by:

$$
\tau=\frac{\nu}{T}+\frac{\Delta t}{2}
$$

The macroscopic quantities, density $\rho$ and momentum density $\rho \mathbf{u}$, are determined directly from the distribution function $F_{i}$, using the moment summations:

$$
\rho(\mathbf{x}, t)=\sum_{i} F_{i}(\mathbf{x}, t), \quad \rho \mathbf{u}(\mathbf{x}, t)=\sum_{i} F_{i}(\mathbf{x}, t) \xi_{i} .
$$

The full compressible viscous Navier-Stokes equations can be recovered from the Lattice Boltzmann equation, using a multi-scale analysis, ${ }^{25}$ for wavelengths $\lambda \gg \Delta x$ and frequencies $f \ll \Delta t$. The resulting equation of state is that of an ideal gas, in the form $P=\rho T$.

The numerical scheme is solved on a grid composed of cubic volumetric elements called voxels, and variable resolution (VR) is allowed, where the grid size changes by a factor of two for adjacent resolution regions. The local computational nature of LBM allows for excellent parallelization, and the method is well adapted to handle complex arbitrary geometries.

Together with the definition of $F_{i}^{e q}$, equations (1) and (2) constitute the LBM scheme used to perform direct numerical simulations (DNS). For high Reynolds number simulations, turbulence modeling is incorporated into the Lattice Boltzmann equations by replacing the relaxation time by an effective turbulent relaxation time scale in equation (2). Additional details on the LBM numerical schemes, and the use of wall boundary conditions and turbulence modeling can be found in Refs. 26-29.

\section{B. Numerical setup}

The CAD geometry of the experimental wing is used to generate the computational mesh. Most of the internal piping for the actuators is removed, as well as the airfoil support, as shown in figure 4(a), and $(b)$. However, the detailed geometry at the leading edge of the wing (i.e., the rim and the end of the actuator pipes) is conserved in order to capture the main features of the actuator flow physics. In the simulations, the pulsed-blowing actuation is imposed as a mass flow boundary condition on the back wall inside of each actuator pipe, as indicated by the red arrow in figure $4(b)$.

In the study presented here, three angles of attack $\alpha=0^{\circ}, 10^{\circ}$ and $19^{\circ}$ are considered. The other simulation parameters match the experimental flow condition, including the freestream velocity $U_{0}=5 \mathrm{~m} / \mathrm{s}$ and the Reynolds number $R e=68000$.

First, a preliminary resolution study is performed to determine the grid size required on the airfoil surface to accurately predict the lift and drag for the natural flow. Resolutions of $1.6 \mathrm{~mm}, 0.8 \mathrm{~mm}$ and $0.4 \mathrm{~mm}$ were considered. The mean forces predicted from the simulations were compared to the experimental results without actuation at $\alpha=10^{\circ}$. A finest resolution of $0.4 \mathrm{~mm}$ (i.e., about 500 grid points per chord length) was found sufficient to insure good accuracy while keeping the computational cost reasonable. This resolution 


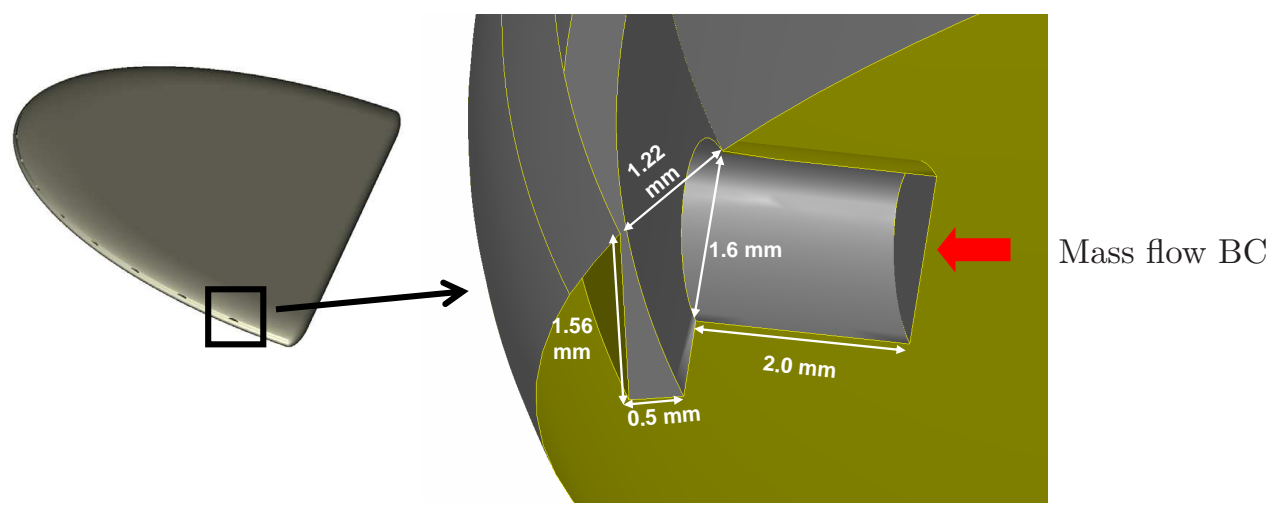

(a)

(b)

Figure 4. Schematic of the airfoil geometry for the LBM simulation: (a) overall view (b) zoomed view of one actuator. The red arrow indicates the location of the mass flow boundary condition (BC) used in the simulation to reproduce the experimental actuation.

leads to only 4-5 cells across the actuator elliptical cross-section. However, without actuation, the flow inside the rim and the actuator pipe is essentially zero. Adding resolution in these elements is therefore not expected to change the results significantly.

In contrast, for the cases with actuation, the need for additional resolution can be anticipated to capture the flow field generated by the actuators. Three different grids are considered here. The first one, referred to as $B 04$, is the baseline grid with a finest resolution of $0.4 \mathrm{~mm}$ discussed in the previous paragraph. The second grid A02 is identical to $B 04$, with an additional resolution level because of the actuation, added locally inside the rim and the actuator pipe, with cell size of $0.2 \mathrm{~mm}$. Similarly, the third grid A01 corresponds to A02 with another added resolution level. In that case, the smallest cell size is therefore $0.1 \mathrm{~mm}$, which corresponds to 16-20 cells across each actuator cross-section. Details about the different grids are summarized in table 1.

\begin{tabular}{ccccc} 
& Grid & Resolution & Nb of Cells & CPU hours \\
\hline Actuation off & B04 & $0.4 \mathrm{~mm}$ & 53 Million & 3000 \\
\hline Actuation on & $B 04$ & $0.4 \mathrm{~mm}$ & 53 Million & 3000 \\
& A02 & $0.2 \mathrm{~mm}$ & 57 Million & 4500 \\
& A01 & $0.1 \mathrm{~mm}$ & 64 Million & 10000
\end{tabular}

Table 1. Grid details and computation costs for 1s of simulated physical time.

It is important to note that the objective here is to have a grid size sufficiently small to supply physical actuation without the need for artificial forcing. It is not to fully resolve the boundary layer details inside the actuators, which would be prohibitively expensive, without much expected benefits. For the same reason, the wall boundary conditions in the rim and actuators are set to frictionless.

Figure 5 shows two cut planes in grid A01, including the 3 VR regions discussed above, to illustrate the type of mesh used in this study. A total of 10 variable resolution regions is used in the simulations. Note that particular care is also taken to have adequate resolution above the airfoil and downstream of the trailing edge, to resolve the separated region and the unsteady wake.

The trailing edge of the airfoil is centered at $(x / c, y / c, z / c)=(0,0,0)$ and the total size of the domain is approximately $(80 c, 65 c, 65 c)$. The upstream boundary condition is an inlet with uniform and constant streamwise velocity $U_{0}$, while the top, bottom, and downstream boundary conditions are pressure outlets $\left(P=P_{0}=1 \mathrm{~atm}\right)$. For all the cases and different grids, the physical time step size is $1.87310^{-6} \mathrm{~s}$ and the total simulated time is about 1s. Additional details about the number of cells and the computation cost are presented in table 1 . For the largest grid, the runtime is approximately 3 days on 128 processors.

First, for each angle of attack, a coarse computation is performed to speed up temporal convergence. These simulations are initialized with the airfoil in a uniform flow $U_{0}$, and zero flow inside the rim and 


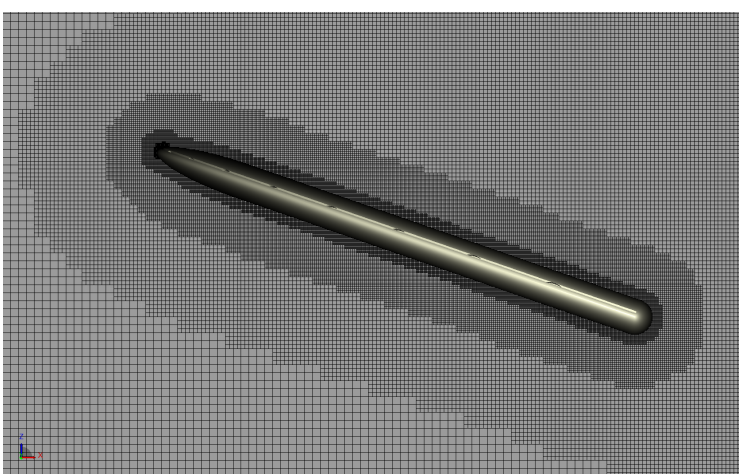

(a)

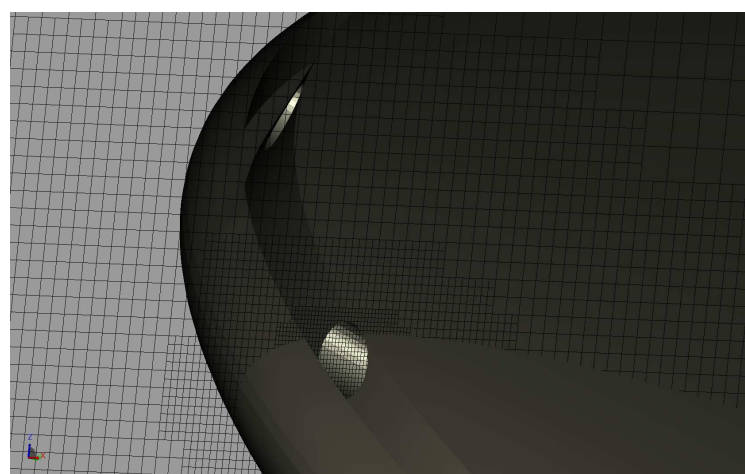

(b)

Figure 5. Resolution details for grid A01: (a) mesh around the airfoil (b) zoomed view of the mesh at one actuator.

the actuator pipes. The first $0.2 \mathrm{~s}$ of data is discarded to remove the initial transient, and the last flow field is saved, after approximately $0.5 \mathrm{~s}$ of computation. For the baseline cases (i.e., without actuation), the simulation is restarted with that last saved flow field as initial condition, and continued for 1s. Similarly for the active flow control cases (i.e., with actuation), the simulation are again restarted from the same saved flow field, using a seeding technique if the grid is changed. The mass flow boundary condition is turned on for $0.017 \mathrm{~s}$. The simulations are then continued with the actuator off for a total duration of $1 \mathrm{~s}$. This procedure yields a clear comparison of the numerical results with and without actuation, and enables a precise calculation of the change in lift $\Delta L$ due to the control as a function of time.

\section{Results and discussion}

\section{A. Baseline cases}

For the three angles of attack, the mean (steady) lift and drag forces are computed, and the values are reported in figure $6(a)$ and $(b)$. Here, only the experimental results without actuation are relevant, for comparison with the numerical prediction. The experimental data with continuous actuation from reference 4 is shown only to illustrate the effect of the active control, as discussed in section II. Good agreement at all three angles of attack is obtained, in particular for the drag coefficient. The lift coefficient is under predicted in the simulation at the higher angles of attack but within the uncertainty in the experimental and numerical data.

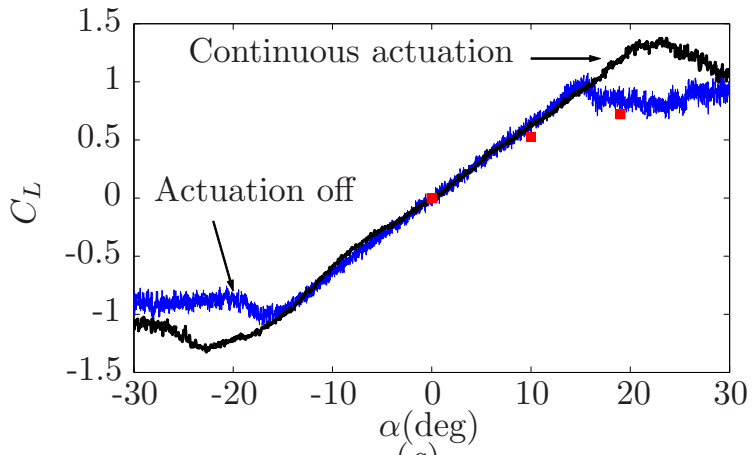

(a)

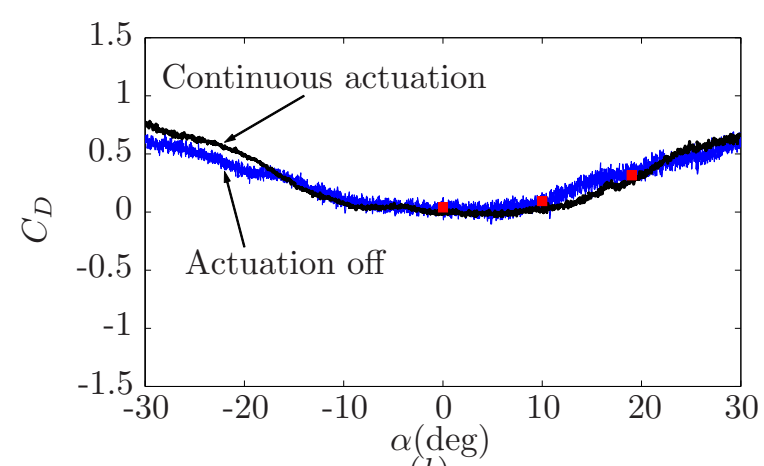

(b)

Figure 6. Steady force coefficients on the wing as a function of the angle of attack: $(a)$ lift; (b) drag. The solid lines correspond to the experimental results and the symbols ( $\square$ ) show the numerical prediction with actuation off.

Visualization of the instantaneous vorticity field in the mid-span plane is presented in figure 7 , for 
$0 \leq|\omega| c / U_{0} \leq 50$. For both $\alpha=0^{\circ}$ and $\alpha=10^{\circ}$ (i.e., pre-stall), the boundary layer clearly remains attached, with periodic shedding from the airfoil trailing edge. As the angle of attack is increased to $\alpha=19^{\circ}$ (i.e., post-stall), the flow over the wing is fully detached, with many fine vortical structures shed from the leading and trailing edge.

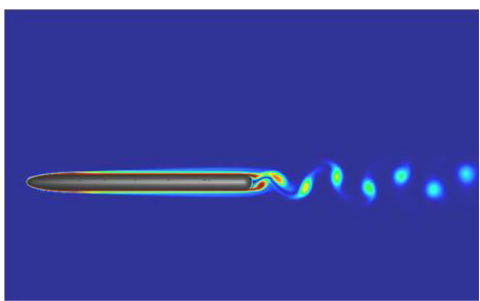

$(a)$

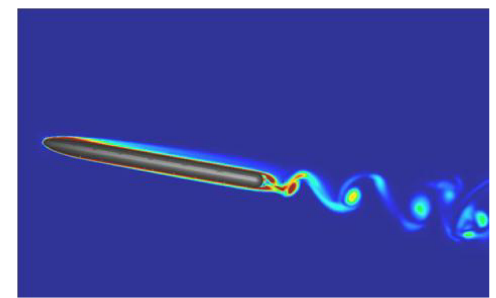

(b)

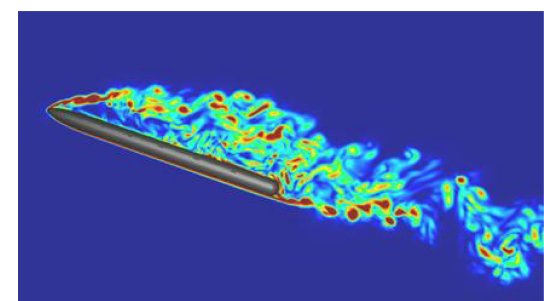

$(c)$

Figure 7. Instantaneous vorticity magnitude in the mid-span plane from the LBM simulation without actuation: (a) $\alpha=0^{\circ} ;\left(\right.$ b) $\alpha=10^{\circ} ;(c) \alpha=19^{\circ}$.

The main features of the time-averaged flow field at angle of attack $\alpha=19^{\circ}$ are presented in figure 8. The streamlines and contours of the velocity magnitude $\left(0 \leq u / U_{0} \leq 1.5\right)$ in the mid-span plane are shown in figure $8(a)$. The flow separation and large recirculation zone above and behind the airfoil can clearly be observed. These features are similar to the experimental results with smoke visualization in figure $3(a)$. Isosurface of the nondimensionalized vorticity magnitude $|\omega| c / U_{0}=2$ is shown in figure $8(a)$. Here the presence of strong tip vortices is reminiscent of the results obtained for similar geometry by Taira and Colonius, at lower Reynolds number. ${ }^{3}$

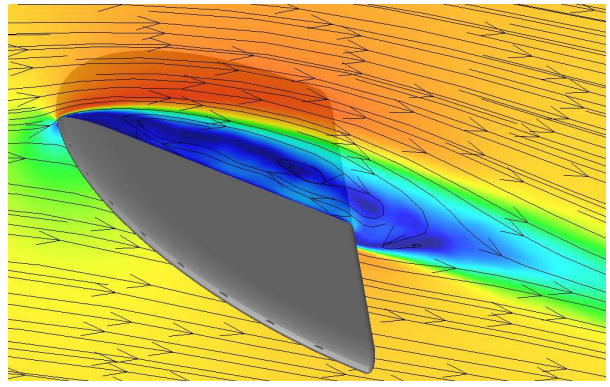

$(a)$

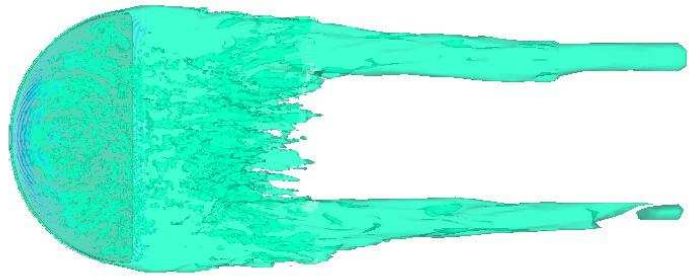

(b)

Figure 8. Mean flow features at $\alpha=19^{\circ}$ without actuation; (a) velocity magnitude and streamlines; (b) isosurface of vorticity magnitude $|\omega| c / U_{0}=2$.

\section{B. Actuation cases}

For all three angles of attack, the single pulsed square wave actuation is turned on for $0.017 \mathrm{~s}$. The resulting jet velocity at the pipe exit is $U_{j e t} \approx 10.9 \mathrm{~m} / \mathrm{s}$, in good agreement with the expected velocity from the experiment. Much like the continuous control shown in figure 6, the single pulse actuation has essentially no effect on the lift and drag for pre-stall angles of attack in the experiment. The same result is observed in the simulations: at $\alpha=0^{\circ}$ and $10^{\circ}$, both transient and mean force coefficients change by less than $1 \%$, with and without control.

In contrast, for $\alpha=19^{\circ}$, a transient increase in lift and drag is measured after the pulse. The time history of the forces on the airfoil is shown in figure 9, for the three grid resolutions. The results for the baseline case (i.e., without actuation) for the same time period is also presented. The start and end of the pulsed square wave actuation can clearly be observed in the time history. Overall, the three grids show the same trends: right after the actuators are turned off, the forces decrease below the baseline value. Then, approximately $0.1 \mathrm{~s}$ after the end of the pulse actuation, both lift and drag increase and remain higher for about $0.5 \mathrm{~s}$, before converging back to the baseline levels. 
For the medium grid A02 and the fine grid A01, the results are similar up to $0.5 \mathrm{~s}$ and the transient increase in lift from the baseline reaches nearly $40 \%$. In contrast, the coarse grid $B 04$ over predicts the initial decrease and under predict the level and length of the transient increase. From these results, the medium grid seems sufficient to capture the main effect of the flow actuation. It is important to note here that the simulation on the fine grid $A 01$ was initially ran for $0.5 \mathrm{~s}$ and then restarted to collect more data. This could potentially explain the different trend observed for the red curve in figure 9 for $t>0.5 \mathrm{~s}$.

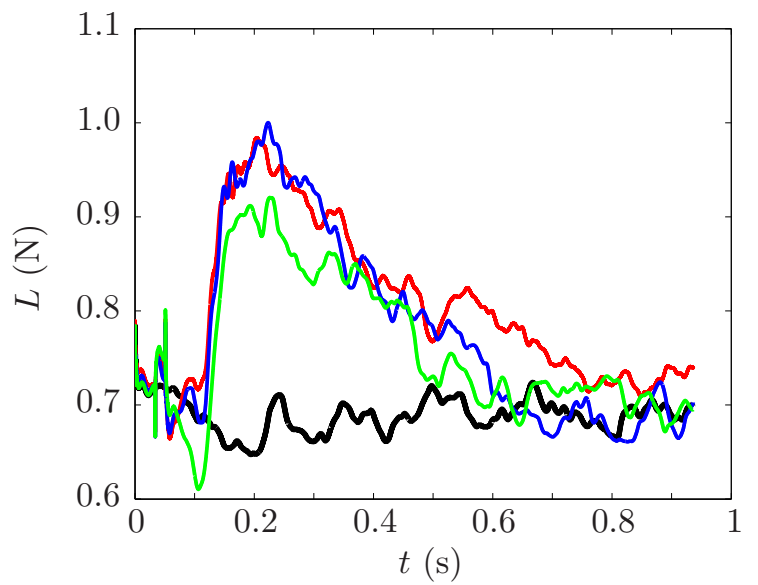

$(a)$

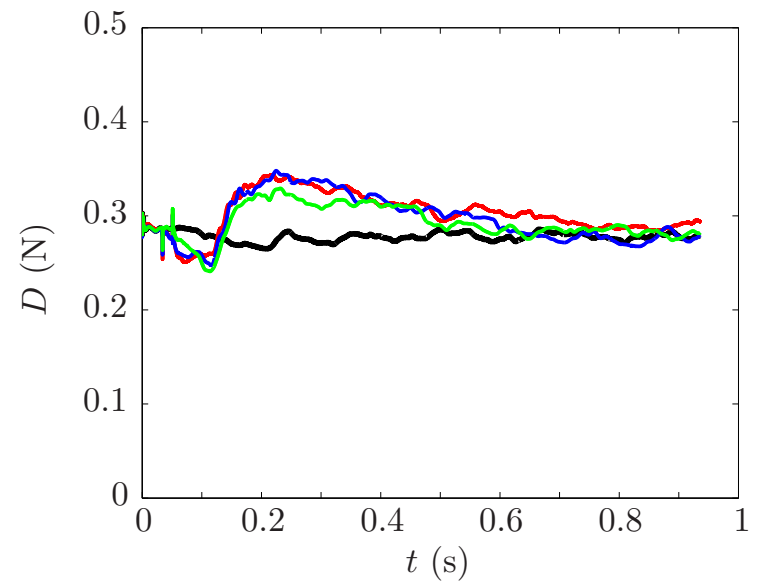

(b)

Figure 9. Time history of the forces on the wing at $\alpha=19^{\circ}$ for the actuation off ( $\longrightarrow$ ), and the actuation

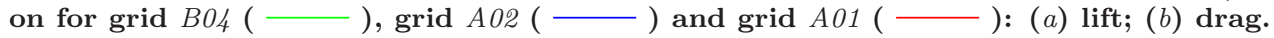

As anticipated, the flow control leads to a significant increase in lift and only a limited increase in drag. To further investigate the influence of the actuation, the transient increase in lift $\Delta L$ is computed as a function of time by taking the difference between lift from the actuated case on grid A02 (blue curve in figure $9(a)$ ) and from the baseline case (black curve in figure 9(a)). The result is compared to the experimental data in figure 10. Here, the numerical result is for one single cycle of $1 \mathrm{~s}$ for the actuation. In contrast, the experimental result corresponds to approximately 60 cycles of $1 \mathrm{~s}$, and is phase-averaged to remove the high frequency fluctuations. Also, in this figure, the simulation time was shifted to align the starts of the pulsed square wave actuation.

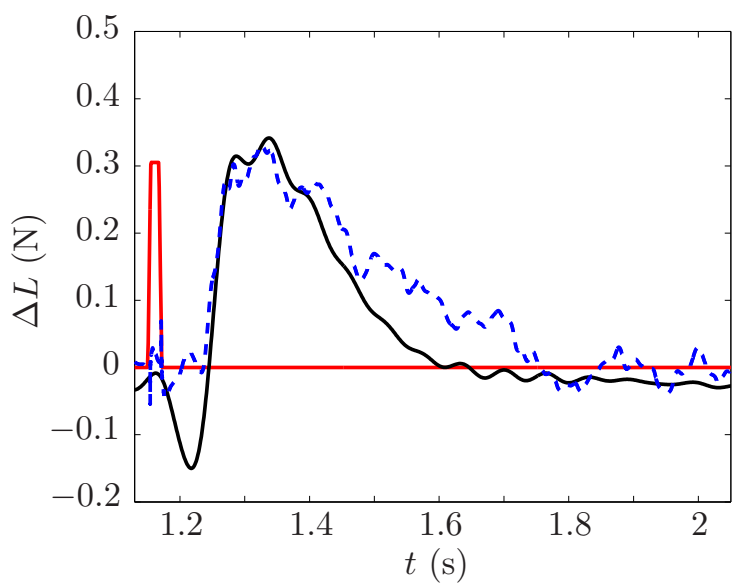

Figure 10. Change in lift $\Delta L$ between actuation on and off at $\alpha=19^{\circ}:(-)$ experiment; ( ----$)$ LBM simulation. The red line ( -$)$ represents the valve voltage and indicates the duration of the pulsed square wave actuation.

Overall, the simulation captures the main feature of the transient increase in lift. The maximum lift is in good agreement but the initial decrease is under predicted. When blowing is initiated, there is a 
short transient when the lift increment is actually negative. This is typically called "non-minimum phase behavior". Similarly, there is a short positive transient lift increment when the blowing is halted. Both behaviors are observed in the experiments, for pulsed and continuous actuations, and seems to be captured in the LBM simulations as well. Further analysis is required to investigate the cause of these short transients.

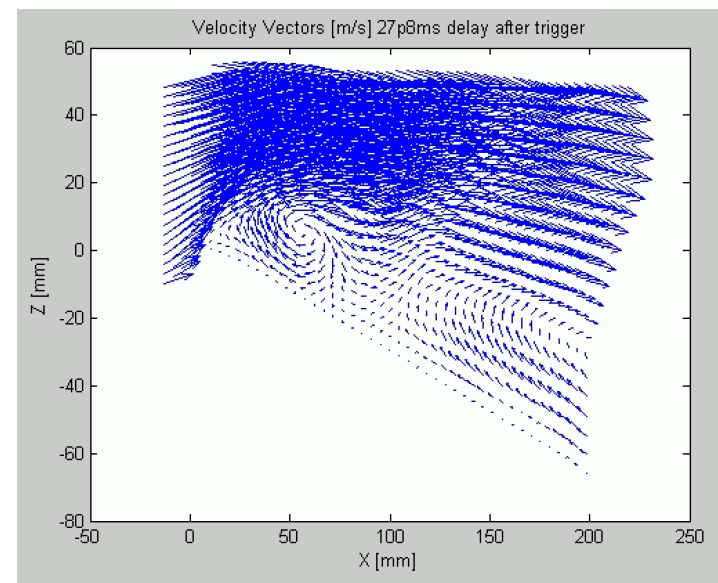

(a)

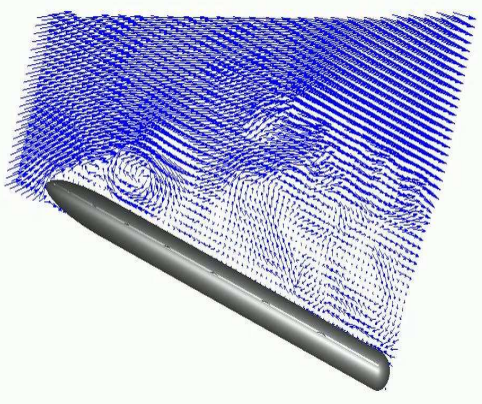

$(b)$

Figure 11. Instantaneous velocity vectors in the mid-span plane: (a) experiment; (b) LBM simulation.

In addition to these features, there is a fairly long time-scale corresponding to the ramp up or ramp down to the baseline lift when forcing is turned on and off. Clearly this timescale is associated with the advection of structures, but seems to take many convective units to die away. To clarify this observation, PIV measurements and numerical results are compared in figure 11. Here, the velocity vectors in the mid-span plane are shown approximately $0.1 \mathrm{~s}$ after the end of the pulse actuation. As mentioned in the previous section and observed in figure 9, this time corresponds to the start of the transient increase in lift. Note that the plot in figure $11(b)$ is stretched to match the format of the experimental data. Overall, good agreement is obtained between the experimental and numerical results. The presence of a large vortex generated by the flow actuation at the leading edge can be observed with both methods. This large vortical structure is then convected downstream, and replaced by smaller vortices shed from the leading edge.

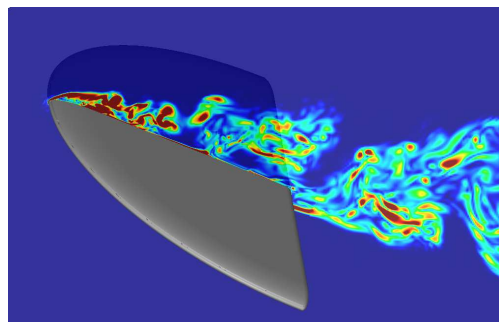

(a)

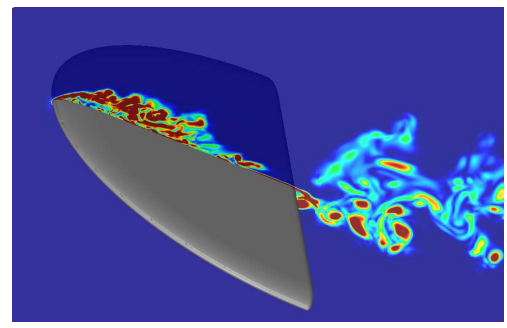

(b)

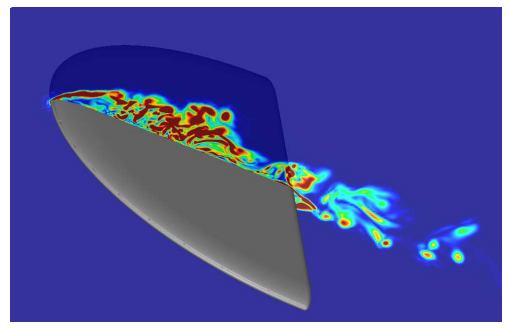

$(c)$

Figure 12. Instantaneous vorticity magnitude in the mid-span plane from the LBM simulation with active flow control, with time $t$ (measured from the end of the pulsed actuation) increasing from left to right: (a) $t=0.05 \mathbf{s} ;(b) t=0.1 \mathbf{s} ;(c) t=0.15 \mathbf{s}$.

Similar conclusions can be obtained from further inspection of the transient numerical results. Three snapshots of the vorticity field for the simulation with actuation, at $\alpha=19^{\circ}$, are presented in figure 12 . This visualization matches the location (mid-span plane) and the vorticity magnitude levels (from 0 to 50 ) from figure 7 . The instantaneous flow field in figure $(a),(b)$, and $(c)$ correspond approximately to time 0.05 $\mathrm{s}, 0.1 \mathrm{~s}$, and $0.15 \mathrm{~s}$ after the end of the pulsed square wave actuation, respectively. From these figures, it 
appears that the main effect of the pulsed actuation is to clear the separated flow off the suction surface of the wing. This process allows for the "regrowth" of a qualitatively more attached boundary layer, resulting in a transient increase in lift. Eventually, the flow over the wing becomes fully detached again, and the lift goes back to the baseline value.

\section{Conclusions and future work}

Numerical simulations of the unsteady flow over a semi-circular planform wing are performed using an unsteady fluid flow solver based on the Lattice Boltzmann method. The model wing used in this study is a low-aspect-ratio, low Reynolds number semicircle airfoil from previous wind tunnel experiments. The LBM simulations exactly match the flow conditions (freestream velocity $U_{0}=5 \mathrm{~m} / \mathrm{s}$, Reynolds number $R e=68000)$ and the detailed geometry in the experiment, including the actuators installed internally along the leading edge of the wing.

Three angles of attack $\alpha=0^{\circ}, 10^{\circ}$ and $19^{\circ}$, with the active flow control both on and off, are investigated. To reproduce the pulsed-blowing actuation used in the experiment, a single pulsed square wave forcing is imposed in the simulations as a mass flow boundary condition in the actuators. The control is turned on for $0.017 \mathrm{~s}$, and the simulations are then continued with the actuator off for a total duration of $1 \mathrm{~s}$.

For both mean and unsteady lift and drag, the numerical simulations show good agreement with the experiments. In particular, the transient increase in lift after the forcing is turned off is well captured in the simulation. Both PIV measurements and transient numerical results indicate that this behavior is associated with the advection of large vortical structures generated by the flow actuation at the leading edge.

Overall, this computational study showed promising results and demonstrate the feasibility of numerical simulations of fully detailed MAV in real flight conditions. Additional simulations will be performed at higher angles of attack (up to $\alpha=30^{\circ}$ ), to investigate the decrease in lift enhancement observed in the experiments with actuation, at these conditions. Future work also includes simulations with different actuator amplitudes, as the experiments showed the maximum in the transient lift response scaled with the peak forcing velocity.

\section{References}

${ }^{1}$ Pines, D. J. and Bohorquez, F., "Challenges Facing Future Micro-Air-Vehicle Development," J. Aircraft, Vol. 43(2), 2006, pp. 290-305.

${ }^{2}$ Torres, G. E. and Mueller, T. J., "Low-Aspect-Ratio Wing Aerodynamics at Low Reynolds Numbers," AIAA J., Vol. 42(5), 2004, pp. 865-873.

${ }^{3}$ Taira, K. and Colonius, T., "Three-dimensional flows around low-aspect-ratio flat-plate wings at low Reynolds numbers," J. Fluid Mech., Vol. 623, 2009, pp. 187-207.

${ }^{4}$ Tadmor, G., Williams, D. R., Collins, J., Colonius, T., and Rowley, C. W., "Control of a Semi-Circular Planform Wing in a "Gusting" Unsteady Free stream Flow II: Modeling and Feedback Design," AIAA Paper 2008-3977, 2008.

${ }^{5}$ Williams, D. R., Collins, J., Tadmor, G., and Colonius, T., "Control of a Semi-Circular Planform Wing in a "Gusting" Unsteady Free stream Flow I: Experimental Issues," AIAA Paper 2008-3976, 2008.

${ }^{6}$ Williams, D. R., Collins, J., Jankhot, C., Colonius, T., and Tadmor, G., "Control of Flow Structure on a Semi-Circular Planform Wing," AIAA Paper 2008-597, 2008.

${ }^{7}$ Williams, D. R., Quach, V., Kerstens, W., Buntain, S., Tadmor, G., Rowley, C., and Colonius, T., "Low-Reynolds Number Wing Response to an Oscillating Freestream with and without Feed Forward Control," AIAA Paper 2009-143, 2009.

${ }^{8}$ Williams, D. R., Tadmor, G., Colonius, T., Kerstens, W., Quach, V., and Buntain, S., "The Lift Response of a Stalled Wing to Pulsatile Disturbances," AIAA J., Vol. in print, 2009.

${ }^{9}$ Qian, Y., d'Humieres, D., and Lallemand, P., "Lattice BGK models for Navier-Stokes Equation," Europhys. Lett., Vol. 17(6), 1992, pp. 479-484.

${ }^{10}$ Chen, H., Teixeira, C., and Molvig, K., "Digital Physics Approach to Computational Fluid Dynamics, Some Basic Theoretical Features," Intl. J. Mod. phys. C, Vol. 8(4), 1997, pp. 675.

${ }^{11}$ Shan, X., Yuan, X., and Chen, H., "Kinetic Theory Representation of Hydrodynamics: a way beyond the Navier-Stokes equation," J. Fluid Mech., Vol. 550, 2006, pp. 413-441.

${ }^{12}$ Shock, R., Mallick, S., Chen, H., Yakhot, V., and Zhang, R., "Recent results on two-dimensional airfoils using a lattice Boltzmann-based algorithm," J. Aircraft, Vol. 39(3), 2002, pp. 434-439.

${ }^{13}$ Fares, E., "Unsteady Flow Simulation of the Ahmed Reference Body using a Lattice Boltzmann Approach," Comput. Fluids, Vol. 35(8-9), 2006, pp. 940-950.

${ }^{14}$ Vaillant, O. and Maillard, V., "Numerical Simulation of Wall Pressure Fluctuation on a Simplified Vehicle Shape," AIAA Paper 2003-3271, 2003.

${ }^{15}$ Pérot, F., Meskine, M., and Vergne, S., "Investigation of the Statistical Properties of Pressure Loadings on Real Automotive Side Glasses," AIAA Paper 2009-3402, 2009. 
${ }^{16}$ Li, Y., Shock, R., Shang, R., and Chen, H., "Numerical Study of Flow Past an Impulsively Started Cylinder by the Lattice-Boltzmann Method," J. Fluid Mech., Vol. 519, 2004, pp. 273-300.

${ }^{17}$ Brès, G. A., Wessels, M., and Noelting, S., "Tandem Cylinder Noise Predictions Using Lattice Boltzmann and Ffowcs Williams - Hawkings Methods," AIAA Paper 2010-3791, 2010.

${ }^{18}$ Ricot, D., Maillard, V., and Bailly, C., "Numerical Simulation of the Unsteady Flow Past a Cavity and Application to Sunroof Buffeting," AIAA Paper 2001-2112, 2001.

${ }^{19}$ Kotapati, R., Keating, A., Kandasamy, S., Duncan, B., Shock, R., and Chen, H., "The Lattice-Boltzmann-VLES Method for Automotive Fluid Dynamics Simulation, a Review," SAE Paper 2009-26-057, 2009.

${ }^{20}$ Keating, A., Beedy, J., and Shock, R., "Lattice Boltzmann Simulations of the DLR-F4, DLR-F6 and Variants," AIAA Paper 2008-749, 2008.

${ }^{21}$ Keating, A., Dethioux, P., Satti, R., Noelting, S., Louis, J., Van de Ven, T., and Vieito, R., "Computational Aeroacoustics Validation and Analysis of a Nose Landing Gear," AIAA Paper 2009-3154, 2009.

${ }^{22}$ Noelting, S., Brès, G. A., Dethioux, P., Van de Ven, T., and Vieito, R., "A Hybrid Lattice-Boltzmann/FH-W Method to Predict Sources and Propagation of Landing Gear Noise," AIAA Paper 2010-3976, 2010.

${ }^{23}$ Wolf-Gladrow, D., Lattice-Gas Cellular Automata and Lattice Boltzmann Models, Springler Verlag, Germany, 2000.

${ }^{24}$ Bhatnagar, P., Gross, E., and Krook, M., "A model for collision progresses in gases. I. small amplitude processes in charged and neutral one-component system," Phys. Rev., Vol. 94(3), 1954, pp. 511-525.

${ }^{25}$ Chapman, S. and Cowling, T., The Mathematical Theory of Non-Uniform Gases, Cambridge University Press, 1990.

${ }^{26}$ Chen, H., Teixeira, C., and Molvig, K., "Realization of Fluid Boundary Conditions via Discrete Boltzmann Dynamics," Intl. J. Mod. phys. C, Vol. 9(8), 1998, pp. 1281.

${ }^{27}$ Chen, H., "Volumetric Formulation of the Lattice Boltzmann Method for Fluid Dynamics: Basic Concept," Phys. Rev. $E$, Vol. 58, 1998, pp. 3955-3963.

${ }^{28}$ Chen, S. and Doolen, G., "Lattice Boltzmann Method for Fluid Flows," Ann. Rev. Fluid Mech., Vol. 30, 1998, pp. 329364.

${ }^{29}$ Chen, H., Orszag, S., Staroselsky, I., and Succi, S., "Expanded Analogy between Boltzmann Kinetic Theory of Fluid and Turbulence," J. Fluid Mech., Vol. 519, 2004, pp. 307-314. 